\title{
ALGORITHMS FOR TESTING THE DIAGONAL SIMILARITY OF MATRICES AND RELATED PROBLEMS*
}

\author{
GERNOT M. ENGEL † AND HANS SCHNEIDER $\dagger$
}

\begin{abstract}
A simple algorithm is presented for testing the diagonal similarity of two square matrices with entries in a field. Extended forms of the algorithm decide various related problems such as the simultaneous dingonal similarity of two families of matrices, the existence of a matrix in a subfield diagonally similar to a given matrix, the existence of a unitary matrix similar to a given complex matrix, and the corresponding problems for diagonal equivalence in place of diagonal similarity. The computational complexity of our principal algorithm is studied, programs and examples are given. The algorithms are based on the existence of a canonical form for diagonal similarity. In the first part of the paper theorems are proved which establish the existence of this form and which investigate its properties.
\end{abstract}

1. Introduction. In this paper we present a simple algorithm for testing the diagonal similarity of two square matrices with entries in a field $\mathbb{F}$. Extended forms of our algorithm decide the simultaneous diagonal similarity of two families of matrices, the existence of a matrix in a subfield diagonally similar to a given matrix and, if $\mathbb{F}$ is the real or complex field, the existence of a real orthogonal or unitary matrix diagonally similar to a given matrix. Another modification of our algorithm tests the diagonal equivalence of two rectangular matrices. There exist extensions for diagonal equivalence which correspond to the extensions described above in the case of diagonal similarity.

After the appropriate definitions $(\S 2)$, we develop the theory on which our algorithm is based ( $\$ 3$ and $\S 4$ ). We show that for $A \in \mathbb{F}^{n n}$, the set of $n \times n$ matrices with elements in $\mathbb{F}$, there exists a canonical form for diagonal similarity. We denote this form by $A_{F}$, since it depends on a choice of a spanning forest $F$ for the graph $G(A)$ of $A$ considered as an undirected multigraph. Further, we give a simple construction for a diagonal matrix $X$ such that $X A X^{-1}=A_{F}$ and we write $X=$ $X(A, F, U)$ since $X$ also depends on a choice of a set of representatives $U$ for the connected components of $F$ or $G(A)$. Thus, for $A, B \in \mathbb{F}^{n n}$, the matrices $A$ and $B$ are diagonally similar if and only if $G(A)=G(B)$ and $A_{F}=B_{F}$ or, equivalently, $H_{F}$ is a $\{0,1\}$ matrix where $H=A \odot B$ is the Hadamard quotient defined in [1] or $\S 3$. Thus we have the following simple procedure to test diagonal similarity of $A$ and $B$ :

(1) Check whether $G(A)=G(B)$.

(2) If so, choose a spanning forest $F$ for $G(A)$ and a set $U$ of representatives for the connected components of $G(A)$.

(3) For $H=A \odot B$, compute $X=X(H, F, U)$.

(4) Check whether $X H X^{-1} \in\{0,1\}^{n n}$.

A detailed description of the algorithm and a study of its computational complexity is given in $\S 5$. In $\S 6$ we briefly indicate applications which are more fully described in our technical report with the same title as this paper.

The relationship between cyclic products and diagonal similarity which is crucial to our theory can be traced back as far as Fiedler-Pták [4]. Theorems with proofs on which algorithms may be based are given in [6], e.g. Theorem 3.17, though no actual

\footnotetext{
* Received by the editors January 30, 1981, and in revised form July 28, 1981.

t 2154 Pinar Place, Del Mar, California 92014.

$\ddagger$ Mathematics Department, University of Wisconsin, Madison, Wisconsin 53706. The research of this author was supported in part by the National Science Foundation under grants MCS 78-01087 and MCS 80-26132 and by the Deutsche Forschungsgemeinschaft (DFG) while he was a Visiting Professor at the Mathematisches Institut der Universität Würzburg, D-8700 Würzburg, West Germany.
} 
algorithm is to be found in that paper. The cycles used in these papers are of a restricted type which occur in the evaluation of determinants; i.e., an arc $(i, j)$ is traversed only from $i$ to $j$. In view of this, unless there is an irreducibility condition on the matrix, any algorithm based on these results requires the determination of the Frobenius block form of the matrix. For the special problem of diagonal similarity to a unitary matrix an interesting algorithm of this type is to be found in.Berman-ParlettPlemmons [1]. The use of general cycles to prove results on diagonal similarity occurs in [6]. Though the proofs in that paper are geometric and existential, it is these features which allow us here to develop constructive proofs and algorithms which do not require the Frobenius block form. The corresponding tool is a spanning forest of an undirected multigraph, which has already been mentioned and which is simple to compute. Thus our algorithm appears to have computational advantages.

\section{Definitions.}

DEFINITION 2.1. Formally, a (simple, directed) graph $G$ is a pair $G=(I, E)$ of finite sets with $E \subseteq I \times I$. The elements of $I$ are called the vertices of $G$, and the elements of $E$ the arcs of $G$. We represent graphs in the usual way, see, e.g., Fig. 1, where $e_{1}=(1,2)$, etc.
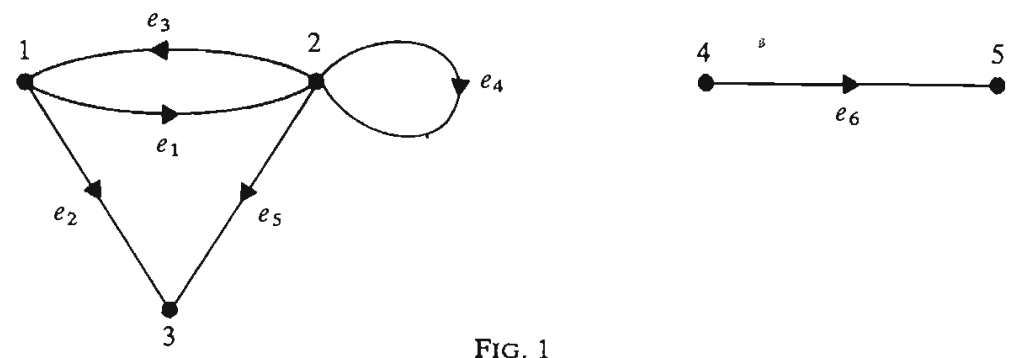

FrG. 1

Since this graph will be used as an example several times, we shall call it $G^{*}$. Although in Fig. 1 we use arrows to represent arcs, we give the symbols $i \rightarrow j$ and $j \leftarrow i$ somewhat different meanings in the text. A link in $G$ is a triple $\lambda=(i, j, \varepsilon)$ where $(i, j) \in E$ and $\varepsilon= \pm 1$.

If $\varepsilon=+1,(\varepsilon=-1)$ we call $i$ the start (end) and $j$ the end (start) of $\lambda$. Intuitively, we may consider $(i, j,+1)$ as the arc $(i, j)$ traversed from $i$ to $j$, and $(i, j,-1)$ as the same arc, traversed from $j$ to $i$. Thus it is natural to represent $(i, j,+1)$ by $i \rightarrow j$ and $(i, j,-1)$ by $j \leftarrow i$.

A chain in $G$ is a sequence $\alpha=\left(\lambda_{1}, \cdots, \lambda_{s}\right)$ of links in $G$ for which the end of $\lambda_{p}$ is the start of $\lambda_{p+1}, p=1, \cdots, s-1$. The start $i$ of $\alpha$ is the start of $\lambda_{1}$, the end $j$ of $\alpha$ is the end of $\lambda_{s}$. We also say that $\alpha$ is a chain from $i$ to $j$. Our notation for links is immediately extended to chains, as we illustrate by means of examples from the graph $G^{*}$ of Fig. 1:

Thus

$$
\alpha=3 \leftarrow 1 \rightarrow 2 \rightarrow 2, \quad \beta=3 \leftarrow 1 \leftarrow 2 \rightarrow 2
$$

respectively stand for the chains

$$
\begin{aligned}
& \alpha=((1,3,-1),(1,2,+1),(2,2,+1)), \\
& \beta=((1,3,-1),(2,1,-1),(2,2,+1))
\end{aligned}
$$

from 3 to 2 . Observe that $\alpha$ traverses the arc $(1,2)$, while $\beta$ traverses the arc $(2,1)$. Thus the concept of chain formalizes the notion of putting a pencil on a vertex of a graph represented as in Fig. 1 and moving it in or against the direction of a sequence arcs to another vertex. 
Let $\alpha=\left(\lambda_{1}, \cdots, \lambda_{s}\right)$ be a chain in $G$. We call $\alpha$ a simple chain if the starts of $\lambda_{1}, \cdots, \lambda_{s}$ are pairwise distinct. We call $\alpha$ a closed chain if the start and end of $\alpha$ coincide. A simple closed chain is called a cycle.

If $\alpha=\left(\lambda_{1}, \cdots, \lambda_{s}\right)$ and $\beta=\left(\lambda_{s+1}, \cdots, \lambda_{s+t}\right)$ are chains such that the end of $\alpha$ coincides with the start of $\beta$ then $\alpha \beta$ denotes the chain $\left(\lambda_{1}, \cdots, \lambda_{s+1}\right)$. If $\lambda=(i, j,+1)$ is a link then $\lambda^{-1}=(i, j,-1)$ and if $\alpha$ is the chain above then $\alpha^{-1}=\left(\lambda_{s}^{-1}, \cdots, \lambda_{1}^{-1}\right)$. It will also be convenient to regard $\varnothing$ as the empty chain from any vertex to itself.

DEFINITION 2.2. A subgraph of $G=(I, E)$ is a graph $G^{\prime}=\left(I^{\prime}, E^{\prime}\right)$ such that $I^{\prime} \subseteq I$, $E^{\prime} \subseteq E$. Wc write $G^{\prime} \subseteq G$. Let $F=\left(I^{\prime}, E^{\prime}\right)$. We call $F$ a forest if $F$ has no cycles. A maximal forest $F$ contained in $G$ is called a spanning forest, viz. $F$ is a forest and if $F^{\prime}$ is a forest for which $F \subseteq F^{\prime} \subseteq G$ then $F^{\prime}=F$. It is well known that every graph $G=(I, E)$ has a spanning forest $F=\left(I^{\prime}, E^{\prime}\right)$ and that $I^{\prime}=I$.

DEFINITION 2.3. A graph $G=(I, E)$ is connected if for each pair of vertices $\{i, j\}$ there is a chain in $G$ from $i$ to $j$. (Observe that a graph with a single vertex is connected since $\varnothing$ is a chain). A maximal connected subgraph of $G$ is called a component of $G$. A connected forest is called a tree, a connected spanning forest of $G$ is called a spanning tree of $G$. The components of a forest are trees.

For example, a component of the graph of $G^{*}$ of Fig. 1 is $G_{2}^{*}=\left(\{4,5\},\left\{e_{6}\right\}\right)$. A spanning forest of this graph has components $G_{1}^{*}=\left(\{1,2,3\},\left\{e_{1}, e_{2}\right\}\right)$ and $G_{2}^{*}$.

Let $G$ be a graph with components $G_{1}, \cdots, G_{l}$. If $i_{p}$ is a vertex of $G_{p}, p=1, \cdots, t$ we call $U=\left\{i_{1}, \cdots, i_{p}\right\}$ a set of representatives for $G$. If $F$ is a spanning forest for $G$, then $U$ is also a set of representatives for $F$. For example $U^{*}=\{1,4\}$ is a set of representatives for ( $G^{*}$ and) the spanning forest $F^{*}$.

If $F$ is a tree and $i, j$ are vertices in $F$, then it is easy to see that there is a unique simple chain in $F$ from $i$ to $j$. If $G$ is a graph, $F$ a spanning tree for $G$ and $e=(i, j)$ an arc of $G$ which is not in $F$, (write $e \in G \backslash F$ ) then there is a unique cycle $\gamma=$ $\left(\lambda, \lambda_{1}, \cdots, \lambda_{s}\right)$ such that $\lambda=(i, j,+1)$ and $\left(\lambda_{1}, \cdots, \lambda_{s}\right)$ is a chain in $F$. We call this cycle the canonical cycle for $e$ with respect to $F$.

3. Main theoretical results. Subsequently, $\mathbb{F}$ will be a field and $\mathbb{F}^{n n}$ the set of all $(n \times n)$ matrices with entries in $\mathbb{F}$.

Definition 3.1. Let $A, B \in \mathbb{F}^{n n}$. Then $A$ is diagonally similar to $B$ if there exists a (nonsingular) diagonal matrix $X$ in $\mathbb{F}^{n n}$ for which $X A X^{-1}=B$.

Definition 3.2. Let $A \in \mathbb{F}^{n n}$. Let $\langle n\rangle=\{1, \cdots, n\}$. We define the graph $G(A)=$ $(I, E)$ of $A$ thus:

$$
I=\langle n\rangle, \quad(i, j) \in E \quad \text { if } a_{i j} \neq 0, \quad i, j=1, \cdots, n .
$$

Definition 3.3. Let $A \in \mathbb{F}^{n n}$ and let $\alpha=\left(\lambda_{1}, \cdots, \lambda_{s}\right)$ be a chain in $G(A)$, where $\lambda_{p}=\left(i_{p}, j_{p}, \varepsilon_{p}\right), p=1, \cdots, s$. Then the chain product $\pi_{\alpha}(A)$ is defined by

$$
\pi_{\alpha}(A)=a_{i_{1} j_{1}}^{\varepsilon_{1}} a_{i_{2} j_{2}}^{\varepsilon_{2}} \cdots a_{i_{s} j_{s}}^{\varepsilon_{s}} \text {. }
$$

If $\varnothing$ is the empty chain, $\pi_{\varnothing}(A)=1$. If $\alpha \beta$ is defined then $\pi_{\alpha \beta}(A)=\pi_{\alpha}(A) \pi_{\beta}(A)$ and $\pi_{\alpha}{ }^{-1}(A)=\pi_{\alpha}(A)^{-1}$. If $\alpha$ is a cycle we call $\pi_{\alpha}(A)$ a cycle product, etc.

Example 3.4. Let

$$
A^{*}=\left[\begin{array}{lllll}
0 & 1 & 2 & 0 & 0 \\
3 & 4 & 5 & 0 & 0 \\
0 & 0 & 0 & 0 & 0 \\
0 & 0 & 0 & 0 & 6 \\
0 & 0 & 0 & 0 & 0
\end{array}\right]
$$

Then $G(A)=G^{*}$. 
Consider the chains $\alpha=3 \leftarrow 1 \rightarrow 2 \rightarrow 2$, and $\beta=3 \leftarrow 1 \leftarrow 2 \rightarrow 2$. Then $\pi_{\alpha}(A)=$ $a_{13}^{-1} a_{12} a_{22}$ and $\pi_{\beta}(A)=a_{13}^{-1} a_{21}^{-1} a_{22}$.

DEFINITION 3.5. Let $A \in \mathbb{F}^{n n}$. Let $F$ be a spanning forest for $G(A)=G$. We define the canonical form $A_{F}=C=C(A, F)$ of $A$ (with respect to $F$ ) thus: For $1 \leqq i, j \leqq n$,

$$
c_{i j}= \begin{cases}0 & \text { if }(i, j) \notin G, \\ 1 & \text { if }(i, j) \in F, \\ \pi_{\gamma}(A) & \text { if }(i, j) \in G \backslash F,\end{cases}
$$

where $\gamma$ is the canonical cycle for $(i, j)$ with respect to $F$.

Definition 3.6. Let $F$ be a spanning forest for the graph $G(A)$, where $A \in \mathbb{F}^{n n}$. Let $U=\left\{i_{1}, \cdots, i_{\text {l }}\right\}$ be a set of representatives for $G(A)$; cf. Definition 2.3. We define a transforming matrix $X=X(A, F, U)$ by $x_{j}=x_{\beta}(A)$, where, for $j$ in the component $G_{p}$ of $G(A)$, we denote by $\beta$ the unique simple chain in $F$ from $i_{p} \in U$ to $j$. (Thus $x_{j}=1$ if $j \in U$, for then $\beta=\varnothing$.) 2.3,

Example 3.7. For the matrix $A^{*}$ of Example 3.4, and $F^{*}$ and $U^{*}$ as in Definition

$$
\begin{gathered}
A_{F}^{*}=\left[\begin{array}{ccccc}
0 & 1 & 1 & 0 & 0 \\
3 & 4 & \frac{5}{2} & 0 & 0 \\
0 & 0 & 0 & 0 & 0 \\
0 & 0 & 0 & 0 & 1 \\
0 & 0 & 0 & 0 & 0
\end{array}\right] . . \\
X^{*}=X\left(A^{*}, F, U\right)\left[\begin{array}{ccccc}
1 & 0 & 0 & 0 & 0 \\
0 & 1 & 0 & 0 & 0 \\
0 & 0 & 2 & 0 & 0 \\
0 & 0 & 0 & 1 & 0 \\
0 & 0 & 0 & 0 & 6
\end{array}\right] .
\end{gathered}
$$

Note that $X^{*} A^{*}\left(X^{*}\right)^{-1}=A_{F}^{*}$. We now prove that this is true in general.

THEOREM 3.8. Let $A \in \mathbb{F}^{n n}$. Let $F$ be a spanning forest for the graph of $G(A)$ and let $U$ be a set of representatives for $G(A)$. If $A_{F}$ is the canonical form of $A$ with respect to $F$, and $X=X(A, F, U)$ is a transforming matrix, then $X A X^{-1}=A_{F}$.

Proof. Let $C=A_{F}$.

(i) If $(i, j) \notin G(A)$, then evidently $c_{i j}=0$.

(ii) Let $(i, j)=e \in F$, say $e \in F_{p}, 1 \leqq p \leqq t$. Let $\beta_{i}, \beta_{j}$ be the unique simple chains from $i_{p}$ to $i$ and $j$ respectively. Then either $\beta_{j}=\beta_{i}(i \rightarrow j)$ or $\beta_{i}=\beta_{j}(j \rightarrow i)$. Hence either $x_{j}=x_{i} a_{i j}$ or $x_{i}=x_{j} a_{i j}^{-1}$. It follows that $x_{i} a_{i j} x_{j}^{-1}=1$.

(iii) Let $(i, j) \in G \backslash F$. Then the vertices $i, j$ belong to a common component $F_{p}$ of $F$. If $\beta_{i}, \beta_{j}$ are defined as above, then we may write $\beta_{i}=\delta \beta_{i}^{\prime}$ and $\beta_{i}=\delta \beta_{j}^{\prime}$, where the chains $\beta_{i}^{\prime}$ and $\beta_{j}^{\prime}$ have no common link. Hence

$$
\pi_{\alpha}(A)=a_{i j} \pi_{\beta_{j}^{\prime}}(A)^{-1} \pi_{\beta_{j}^{\prime}}(A)=a_{i j} \pi_{\beta_{j}}(A)^{-1} \pi_{\beta_{l}}(A)=x_{i} a_{i j} x_{j}^{-1} .
$$

The matrix $A_{F}$ is indeed a canonical form for $A$ under diagonal similarity. This will be shown in the next corollary.

COROLlaRy 3.9. Suppose that $A, B \in \mathbb{F}^{n n}$. Let $F$ be a spanning forest for $G(A)$. Then the following are equivalent.

(i) $A$ is diagonally similar to $B$,

(ii) $G(A)=G(B)$ and $A_{F}=B_{F}$.

Proof. (ii) $\Rightarrow$ (i). By Theorem 3.8, $A$ is diagonally similar to $A_{F}$ and $B$ is diagonally similar to $B_{F}$. Hence $A$ is diagonally similar to $B$. 
(i) $\Rightarrow$ (ii). Let $A$ be diagonally similar to $B$. Evidently $G(A)=G(B)$ and $\pi_{\gamma}(A)=$ $\pi_{\gamma}(B)$ for all cycles $\gamma$ in $G$. Hence by definition of $A_{F}$, it follows that $A_{F}=B_{F}$.

We state our next corollary in terms of the Hadamard quotient $A \odot B$ of two matrices $A, B$; cf. [1].

Definition 3.10. Let $A, B \in \mathbb{F}^{n n}$ and suppose that $\mathcal{G}(A)=G(B)$. Then the Hadamard quotient $H=A \odot B$ is defined by

$$
h_{i j}= \begin{cases}a_{i j} / b_{i j} & \text { if }(i, j) \in G(A), \\ 0 & \text { otherwise. }\end{cases}
$$

It is clear that $Y A Y^{-1}=B$ is equivalent to $Y(A \odot B) Y^{-1} \in\{0,1\}^{n n}$. Also, a $\{0,1\}$ matrix is in canonical form. Thus, we obtain our chief theoretical tool as an immediate application of Corollary 3.9 .

Corollary 3.11. Let $A, B \in \mathbb{F}^{n n}$. Let $F$ be a spanning forest for $G(A)$, with $U$ a set of representatives for $G(A)$. The following are equivalent.

(i) $A$ is diagonally similar to $B$.

(ii) $G(A)=G(B)$ and $A \odot B$ is diagonally similar to a $\{0,1\}$-matrix.

(iii) $G(A)=G(B)$ and $(A \odot B)_{F}$ is a $\{0,1\}$-matrix.

(iv) $G(A)=G(B)$ and if $X=X(A \odot B, F, U)$ then $X A X^{-1}=B$.

Our algorithm is based on the equivalence of (i) and (iv) of the above theorem. It rests on the computation of $X=X(A \odot E, F, U)$ and $X(A \odot B) X^{-1}$. Even though there may be other diagonal matrices $Y$ such that $Y A Y^{-1}=B$, we emphasize that either $X A X^{-1}=B$ or else $A$ is not diagonally similar to $B$. We now determine those $Y$ for which $Y A Y^{-1}=B$.

THEOREM 3.12. Let $A, B \in \mathbb{F}^{n \mu}$. Let $F$ be a spanning forest for $G(A)$ with components $F_{1}, \cdots, F_{1}$ and let $U$ be a set of representatives for $G(A)$. Let $Y \in \mathbb{F}^{n \mathrm{n}}$ be diagonal. The following are equivalent.

(i) $Y A Y^{-1}=B$.

(ii) $G(A)=G(B)$ and, for $i \in F_{p}$ and $i_{p} \in U, y_{i}=y_{i_{p}} x_{i}$, where $X=X(A \odot B, F, U)$.

Proof. (ii) $\Rightarrow$ (i). Suppose $j \notin F_{p}$. Then $a_{i j}=0$ hence also $b_{i j}=0$ and so $y_{i} a_{i j} y_{j}^{-1}=b_{i j}$. If $j \in F_{p}$, then $y_{i} a_{i j} y_{j}^{-1}=y_{i_{p}} x_{i} a_{i j} x_{j} y_{i_{p}}^{-1}=x_{i} a_{i j} x_{j}^{-1}=b_{i j}$.

(i) $\Rightarrow$ (ii). Evidently $G(A)=G(B)$. Since $i \in F_{p}$, there exists a simple chain $\gamma$ from $i_{p}$ to $i$. Let $H=A \odot B$. Then $Y H Y^{-1}=X H X^{-1}$. Hence $y_{i_{p}} \pi_{\gamma}(H) y_{i}^{-1}=\pi_{\gamma}\left(Y H Y^{-1}\right)=$ $\pi_{\gamma}\left(X H X^{-1}\right)=x_{i_{p}} \pi_{\gamma}(H) x_{i}^{-1}$. But $x_{i_{p}}=1$ and $x_{i}=\pi_{\gamma}(H)$. Hence $y_{i}=y_{i_{p}} x_{i}$.

Corollary 3.13. Let $A, B \in \mathbb{F}^{n n}$ and suppose that $A$ is diagonally similar to $B$. Let $F$ be a spanning forest for $G(A)$ and $U$ a set of representatives for $G(A)$. Then $X=X(H, F, U)$, where $H=A \odot B$, is the unique matrix which satisfies $X A X^{-1}=B$ and $x_{i}=1$ for $i \in U$.

The impact of Corollary 3.13 is this. If $A$ is diagonally similar to $B$, then the matrix $X$ which is given by our algorithm and which satisfies $X A X^{-1}=B$ is in fact independent of the choice of the spanning forest $F$. Another immediate corollary to Theorem 3.12 is the following result, proved by a different method in [6, Prop. 2.3].

COROLLARY 3.14. Let $A, B \in \mathbb{F}^{n H}$ and suppose that $A$ is diagonally similar to $B$. Then the following are equivalent.

(i) $Y A Y^{-1}=B$ implies that $Y=c X(A \odot B, F, U)$ where $0 \neq c \in \mathbb{F}$.

(ii) $G(A)=G(B)$ is connected.

\section{Applications.}

\subsection{Simultaneous diagonal similarity.}

Definition 4.1. Let $P$ be an index set, and let $A^{(p)}, B^{(p)} \in \mathbb{F}^{n n}$, for $p \in P$. Then the families $\left\{A^{(p)}: p \in P\right\},\left\{B^{(p)}: p \in P\right\}$ are simultaneously diagonally similar if there is a diagonal matrix $X \in \mathbb{F}^{n h}, X A^{(p)} X^{-1}=B^{(p)}$, for all $p \in P$. 
Our algorithm can easily be adapted to test for the simultaneous diagonal similarity of finite families of matrices. There is no difficulty in proving the underlying theorem when the index set $P$ is infinite.

Definition 4.2. Let $\left\{H^{(p)}: p \in P\right\}$ be a family of matrices in $\mathbb{F}^{n n}$. We call the family semiconstant if it satisfies the following condition:

For $q, p \in P$, and $1 \leqq i, j \leqq n$ either $h_{i j}^{(p)}=h_{i j}^{(q)}$ or $h_{i j}^{(p)} h_{i j}^{(q)}=0$.

In this case the supremum matrix $S=S\left(H^{(p)}: p \in P\right)$ is defined thus:

For $i, j=1, \cdots, n$,

$$
s_{i j}= \begin{cases}h_{i j}^{(p)} & \text { if there exists } p \in P \text { for which } h_{i j}^{(p)} \neq 0 \\ 0 & \text { otherwise. }\end{cases}
$$

THEOREM 4.3. Let $\left\{A^{(p)}: p \in P\right\},\left\{B^{(p)}: p \in P\right\}$ be two families of matrices in $\mathbb{F}^{n n}$. Then the following are equivalent.

(i) The families are simultaneously diagonally similar.

(ii) (a) $G\left(A^{(p)}\right)=G\left(B^{(p)}\right)$, for $p \in P$.

(b) If $H^{(p)}=A^{(p)} \odot B^{(p)}$, then $\left\{H^{(p)}: p \in P\right\}$ is a semiconstant family of matrices.

(c) Let $S=S\left(H^{p}: p \in P\right\}$ be the corresponding supremum matrix, and let $F$ be a spanning forest of $G(S)$. Then the canonical form $S_{F} \in\{0,1\}^{n n}$.

Proof. (ii) $\Rightarrow$ (i). Let $X S X^{-1} \in\{0,1\}^{n n}$. Then either $s_{i j}=x_{j} x_{i}^{-1}$ or $s_{i j}=0, i, j=$ $1, \cdots, n$. Hence, for each $p \in P, h_{i j}^{(p)}=x_{j} x_{i}^{-1}$ or $h_{i j}^{(p)}=0, i, j=1, \cdots, n$. It follows that $b_{i j}^{(p)}=x_{i} a_{i j}^{(p)} x_{j}^{-1}, i, j=1, \cdots, n$.

(i) $\Rightarrow$ (ii). Evidently $G\left(A^{(p)}\right)=G\left(B^{(p)}\right)$, for $p \in P$. By assumption there exists a diagonal $Y \in \mathbb{F}^{n n}$ for which $Y A^{(p)} Y^{-1}=B^{(p)}$, for $p \in P$. Hence $Y H^{(p)} Y^{-1}=\{0,1\}^{n n}$, for $p \in P$. Thus either $h_{i j}^{(p)}=y_{j} y_{i}^{-1}$ or $h_{i j}^{(p)}=0,1 \leqq i, j \leqq n$. Hence $\left\{H^{(p)}: p \in P\right\}$ is semi-constant. Let $S$ be the corresponding supremum matrix. It follows that $Y S Y^{-1} \in$ $\{0,1\}$, but then $S_{F}\left(Y S Y^{-1}\right)_{F} \in\{0,1\}^{n n}$.

In order to test whether $S_{F} \in\{0,1\}^{n n}$, we need merely to construct a transforming matrix $X=X(S, F, U)$. Hence we have an effective test for simultaneous diagonal similarity.

4.2. Diagonal similarity to a matrix with elements in a subgroup. It is easily seen that all our previous results hold when $\mathbb{F}$ is a (multiplicative) Abelian group with 0 , viz. $\mathbb{F} \backslash\{0\}$ is an Abelian group and $0 c=0=c 0$ for all $c \in \mathbb{F}$. In our next theorem we shall explicitly assume that $\mathbb{F}$ is an Abelian group with 0 and $\mathbb{F}_{1}$ will be a subgroup with 0 . As an example, $\mathbb{F}$ can be chosen to be a field and $\mathbb{F}_{1}$ a subfield, e.g., $\mathbb{F}$ is the real field and $\mathbb{F}_{1}$ the rational field. In another important example $\mathbb{F}$ consists of the reals (rationals) and $\mathbb{F}_{1}$ of the nonnegative reals (rationals).

THEOREM 4.4. Let $\mathbb{F}$ be an Abelian group with 0 and let $\mathbb{F}_{1}$ be a subgroup with 0 . Let $A \in \mathbb{F}^{n}$. Then the following are equivalent.

(i) For some diagonal matrix $X \in \mathbb{F}^{n n}, X A X^{-1} \in \mathbb{F}_{1}^{n n}$.

(ii) $A_{F} \in \mathbb{F}_{1}^{n n}$.

Proof. (ii) $\Rightarrow$ (i). Trivial, since $A_{F}=\left(X A X^{-1}\right)_{F}$.

(i) $\Rightarrow$ (ii). Suppose that $X A X^{-1} \in \mathbb{F}_{1}^{n n}$. Then for every cycle $\gamma$ of $G(A)$ we have $\pi_{\gamma}\left(A_{F}\right)=\pi_{\gamma}(A)=\pi_{\gamma}\left(X A X^{-1}\right) \in \mathbb{F}_{1}$. Since $0,1 \in \mathbb{F}_{1}$, it follows that $A_{F} \in \mathbb{F}_{1}^{n n}$.

At this point it is appropriate to state an easy result that will be used in $\S 4.3$. With the notation of Theorem 4.4 , we observe that $H \in \mathbb{F}_{1}^{n n}$ implies that $X=$ $X(H, F, U) \in \mathbb{F}_{1}^{n n}$. The rest of the proof follows from Corollary 3.11 .

THEOREM 4.5. Let $\mathbb{F}$ and $\mathbb{F}_{1}$ be defined as in Theorem 4.4. Let $A, B \in \mathbb{F}^{n n}$. Then the following are equivalent. 
(i) There is a diagonal matrix $Y \in \mathbb{F}_{1}^{n n}$ for which $Y A Y^{-1}=B$.

(ii) (a) $G(A)=G(B)$.

(b) $(A \odot B)_{F} \in\{0,1\}^{n n}$.

(c) $A \odot B \in \mathbb{F}_{1}^{n n}$.

(iii) Conditions (ii) (a), (b) hold and

(c) $X(A \odot B, F, U) \in \mathbb{F}_{1}^{n n}$ where, as usual, $F$ is a spanning forest for $G(A)$ and $U$ a set of representatives for $G(A)$.

Thus our algorithm tests, for example, if two real matrices are similar by means of a diagonal matrix with positive diagonal entries.

4.3. Diagonal similarity to a unitary matrix. We now prove results for real or complex matrices related to those in [1]. We shall give necessary and sufficient conditions for a complex matrix to be diagonally similar via a complex diagonal similarity to a unitary matrix and for a real matrix to be diagonally similar via a real similarity to an orthogonal matrix. Our results can be stated as one theorem, since a unitary matrix with real entries is of course orthogonal. We call a matrix $Y$ nonnegative if all its entries are nonnegative and we write $Y \geqq 0$.

THEOREM 4.6. Let $\mathbb{F}=\mathbb{R}$ or $\mathbb{F}=\mathbb{C}$, and let $A \in \mathbb{F}^{n n}$. Then the following are equivalent.

(i) There exists a unitary matrix $B \in \mathbb{F}^{n n}$ such that $A$ and $B$ are diagonally similar.

(ii) (a) $A$ is nonsingular.

(b) $Y A^{-1} Y^{-1}=A^{*}$, for some diagonal $Y \in \mathbb{F}^{n n}$ where $Y \geqq 0$.

Proof. (i) $\Rightarrow$ (ii). Let $B=Z A Z^{-1}$ be unitary. Then (ii) (a) evidently holds. Let $Y=Z^{*} Z$. Then $Y \geqq 0$. Since $Z A^{-1} Z^{-1}=\left(Z A Z^{-1}\right)^{-1}=B^{-1}=B^{*}=\left(Z A Z^{-1}\right)^{*}=$ $\left(Z^{-1}\right)^{*} A^{*} Z^{*}$ it follows that $Y A^{-1} Y^{-1}=A^{*}$.

(ii) $\Rightarrow$ (i). Let $Z \in \mathbb{F}^{n n}$, where $Z$ is diagonal and satisfies $Z Z^{*}=Y$. It is easily checked that $Z A Z^{-1}$ is unitary.

By combining Theorems 4.5 and 4.6 we obtain a corollary on which an algorithm may be based.

COROllary 4.7. Let $\mathbb{F}=\mathbb{R}$ or $\mathbb{F}=\mathbb{C}$, and let $A \in \mathbb{F}^{n n}$. Then the following are equivalent.

(i) There exists a unitary matrix $B \in \mathbb{F}^{n n}$ such that $A$ and $B$ are diagonally similar.

(ii) (a) $A$ is nonsingular.

(b) $G\left(A^{-1}\right)=G\left(A^{*}\right)$.

(c) If $X=X\left(A^{-1} \oplus A^{*}, F, U\right)$, then $X \geqq 0$ and $X A^{-1} X^{-1}=A^{*}$.

The nonnegativity condition in (ii) (c) cannot be omitted in the above. For let $\mathbb{F}=\mathbb{R}$, and let $a, b \in \mathbb{F}$ be positive numbers with $a^{2}-b^{2}=1$. Let

$$
A=\left[\begin{array}{ll}
a & b \\
b & a
\end{array}\right] \text {. }
$$

Then $X A^{-1} X=A^{*}$ where $X=\operatorname{diag}(1,-1)$, so that all other conditions in (ii) are satisfied. But every real orthogonal matrix is of the form

$$
C=\left[\begin{array}{rr}
c & d \\
-d & c
\end{array}\right]
$$

with $c^{2}+d^{2}=1$. Let $\gamma$ be the cycle $1 \rightarrow 2 \rightarrow 1$. Then $\pi_{y}(A)>0$ and $\pi_{y}(C) \leqq 0$. Hence $A$ cannot be diagonally similar to a real orthogonal matrix. The matrix $A$ is diagonally similar to the complex orthogonal (not unitary) matrix

$$
B=\left[\begin{array}{cc}
c & -i d \\
i d & c
\end{array}\right]
$$


Indeed $X A X^{-1}=B$ where $X=\operatorname{diag}(1, i)$. The results of this subsection hold for all fields $\mathbb{F}$ with involution, viz. with an automorphism $a-\bar{a}$. The elements in $\mathbb{F}$ of form $q \bar{q}$ play the role of the nonnegative elements.

4.4. Diagonal equivalence. Let $A, B \in \mathbb{F}^{n n^{\prime}}$, the set of $n \times n^{\prime}$ matrices. We call $A$ diagonally equivalent to $B$ if there exists a (nonsingular diagonal $X \in \mathbb{F}^{n n}, Y \in \mathbb{F}^{n^{\prime} n^{\prime}}$ for which $X A Y^{-1}=B$.

For $A \in \mathbb{F}^{n n^{\prime}}$ let

$$
A^{+}=\left[\begin{array}{cc}
0_{n n} & A \\
0_{n^{\prime} n} & 0_{n^{\prime} n^{\prime}}
\end{array}\right] \in \mathbb{F}^{n+n^{\prime}, n+n^{\prime}}
$$

where the orders of the 0 matrices are indicated by subscripts. Let $B \in \mathbb{F}^{n n^{\prime}}, X \in \mathbb{F}^{n n}$, $Y \in \mathbb{F}^{n^{\prime} n^{\prime}}$, where $X, Y$ are diagonal. As observed in $\left[6\right.$, p. 212], $X A Y^{-1}=B$ if and only if $Z A^{+} Z^{-1}=B^{+}$, where $Z=X \oplus Y$. It follows that our theorems have analogues for diagonal equivalence. The graph $G\left(A^{+}\right)$is in fact the bipartite graph of $A$; cf. [6]. It follows that our algorithm can easily be extended to test for the diagonal equivalence of pairs of matrices in $\mathbb{F}^{n n^{\prime}}$, the simultaneous diagonal equivalence of two families of matrices, diagonal equivalence to a matrix in a subfield and diagonal equivalence to unitary matrix, see $[1, T h m .1]$. Since only 0 -elements are introduced in going from $A$ to $A^{+}$the algorithms for diagonal equivalence are of the same complexity as those for diagonal similarity. Further theoretical details are omitted.

5. The principal algorithm. Figure 2 is a structured narrative description of an algorithm to calculate the canonical form and transformation matrix of Definitions 3.5 and 3.6. Figure 3 is a computer implementation of this algorithm in APLGOL computer language [5].

Numbers are placed on the left-hand side of corresponding steps in the two listings.

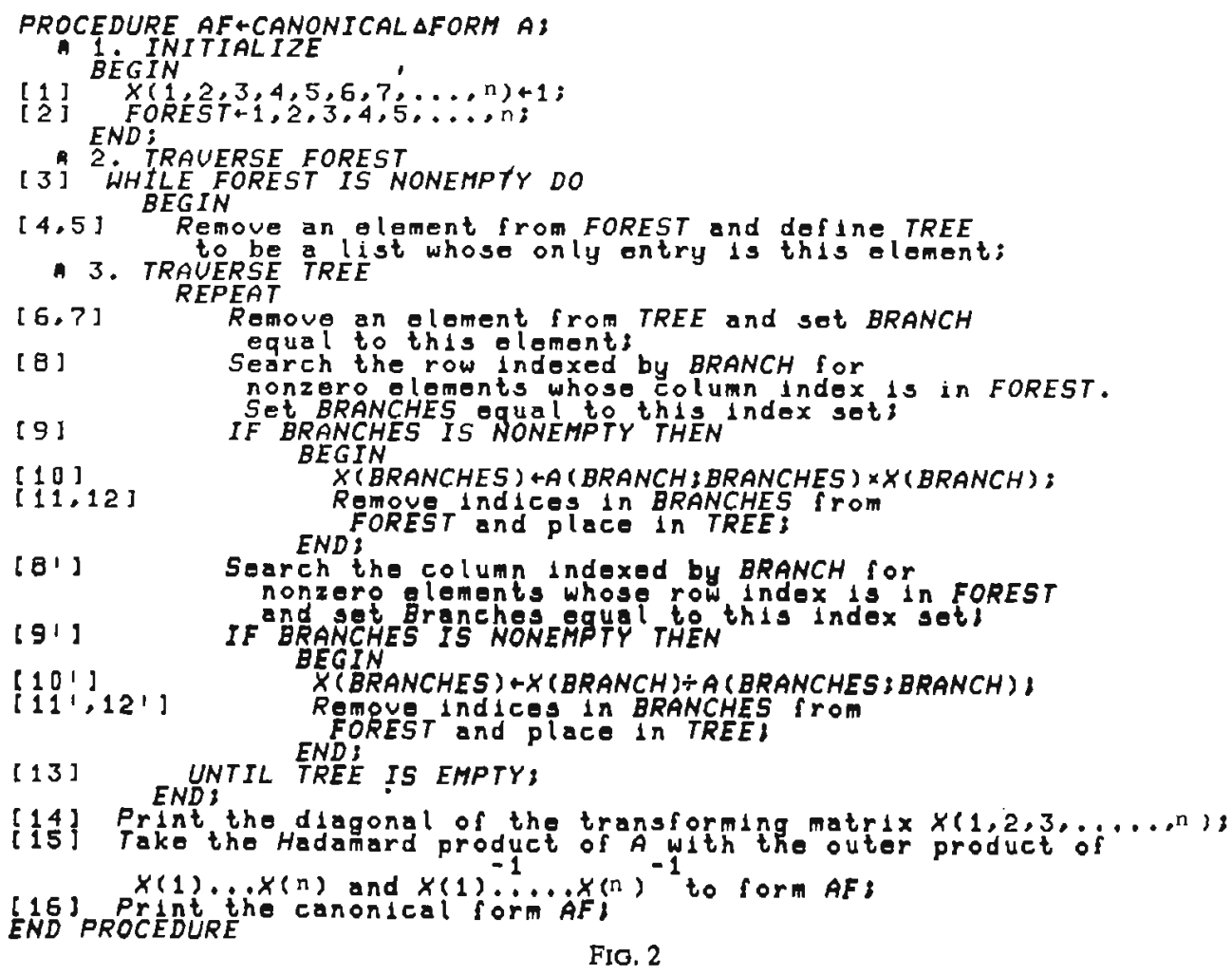

FIG. 2 


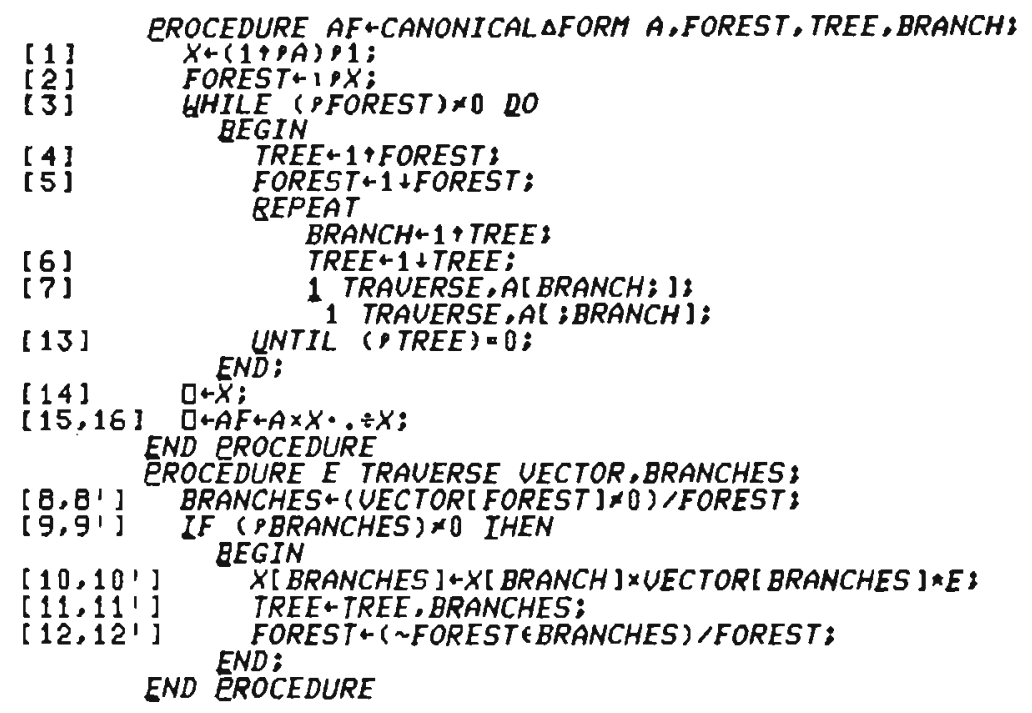

FIG. 3

Computational complexity. If $A$ is a $n \times n$ matrix such that $G(A)$ has $t$ components then the execution of this algorithm results in $6 n-2 t$ storage operations, $n-t$ multiplications or divisions, and fewer then $2 n+t+n^{2}$ but more then $4 n+t-1$ logical operations. Table 1 provides a statement by statement accounting of the complexity.

Steps 1 and 2 are not included in this accounting since the vectors $X$ and FOREST can be initialized prior to execution.

Logical operations are simplified by avoiding the concepts used in analyzing directed graphs. The algorithm involves only straightforward pointer maintenance. Backtracking and recursive executions is avoided. In addition this algorithm does not require precomputation of the column numbers of the nonzeros in each row as is the case in many algorithms in combinatorial matrix theory, e.g., the Duff-Reid implementation of Tarjan's algorithm for the block triangulization of a matrix [2].

TABLE 1

\begin{tabular}{lccc}
\hline $\begin{array}{c}\text { Statement } \\
\text { number }\end{array}$ & $\begin{array}{c}\text { Number of } \\
\text { storage operations }\end{array}$ & $\begin{array}{c}\text { Number of } \\
\text { multiplications } \\
\text { or divisions }\end{array}$ & $\begin{array}{c}\text { Number of logical } \\
\text { operations }\end{array}$ \\
\hline$(3)$ & & & $t$ \\
$(4)$ & $t$ & & \\
$(5)$ & $n$ & & $n$ \\
$(6)$ & $n$ & $n-t$ & $2 n$ \\
$(7)$ & $n$ & & \\
$(13)$ & $n-t$ & & $n-1 \leqq \operatorname{logical}$ op $\leqq n X n-n$ \\
$\left(9,9^{\prime}\right)$ & $n-t$ & & \\
$\left(10,10^{\prime}\right)$ & $n-t$ & $n-t$ & $4 n+t-1 \leqq \operatorname{logical}$ op $\leqq 2 n+t+n^{2}$ \\
$\left(11,11^{\prime}\right)$ & $n-t$ & & \\
$\left(12,12^{\prime}\right)$ & $\left(8,8^{\prime}\right)$ & & \\
\hline Total & $6 n-2 t$ & & \\
\hline
\end{tabular}


6. Applications. We have applied the algorithm of $\S 5$ for finding the canonical form under diagonal similarity to yield the tests shown in Table 2.

TABLE 2

\begin{tabular}{lll}
\hline \multicolumn{1}{c}{ Test } & Justification \\
\hline $\begin{array}{l}\text { Diagonal similarity of a pair of matrices } \\
\text { Simultaneous diagonal similarity of a }\end{array}$ & Corollary 3.11 \\
family of matrices & Theorem 4.3 \\
$\begin{array}{l}\text { Diagonal similarity of a real matrix to } \\
\text { an orthogonal matrix }\end{array}$ & Corollary 4.7 \\
$\begin{array}{l}\text { Extensions of the three algorithms } \\
\text { above to the corresponding algorithms } \\
\text { for diagonal equivalence }\end{array}$ & $\$ 4.4$ \\
\hline
\end{tabular}

The first of these algorithms is described in Fig. 4 in APLGOL notation. Detailed descriptions of some other algorithms are contained in the authors' technical report.

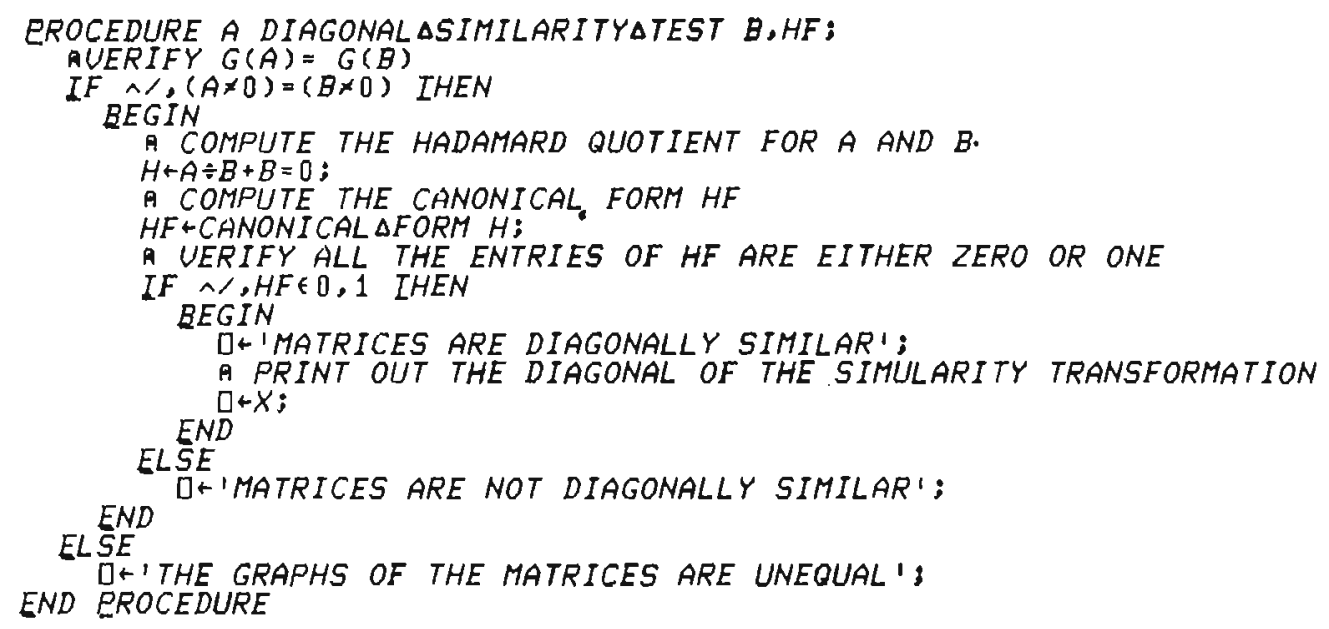

FIG. 4

\section{REFERENCES}

[1] A. Berman, B. N. Parlett and R. J. Plemmons, Diagonal scaling to an orthogonal matrix, this Journal, 2 (1981), pp. 57-65.

[2] I. S. DUFF AND J. K. REID, An implementation of Tarjan's algorithm for the block triangularization of a matrix, ACM Trans. Math. Software, 4 (1978), pp. 137-147.

[3] G. M. ENGEL AND H. SCHNEIDER, Cyclic and diagonal products on a matrix, Linear Alg. Appl., 7 (1973), pp. 301-335.

[4] M. FIEDLER AND V. PTAK, Cyclic products and an inequality for determinants, Czechoslovak Math. J., 19 (1969), pp. 428-450.

[5] R, Kelly AND S, J, WALTERS, APLGOL-2, A structured programming system for APL, IBM Palo Alto Sci. Rep. G 320-3318, 1973.

[6] D. B. SAUNDERS AND H. SCHNEIDER, Flows on graphs applied to diagonal similarity and diagonal equivalence for matrices, Discrete Math., 24 (1978), pp. 205-220. 